\title{
Tumor associated endothelial expression of B7-H3 predicts survival in ovarian carcinomas
}

Xingxing Zang ${ }^{1, *, \dagger}$, Peggy S Sullivan ${ }^{2, \dagger}$, Robert A Soslow ${ }^{3, \dagger}$, Rebecca Waitz ${ }^{1}$, Victor E Reuter ${ }^{3}$, Andrew Wilton ${ }^{4}$, Howard T Thaler ${ }^{4}$, Manonmani Arul ${ }^{5}$, Susan F Slovin ${ }^{5}$, Joyce Wei $^{1}$, David R Spriggs ${ }^{5}$, Jakob Dupont ${ }^{5, \ddagger}$ and James P Allison ${ }^{1}$

${ }^{1}$ Howard Hughes Medical Institute, Immunology Program, Ludwig Center for Cancer Immunotherapy, Memorial Sloan-Kettering Cancer Center, New York, NY, USA; ${ }^{2}$ Department of Pathology and Lab Medicine, David Geffen School of Medicine at UCLA, Los Angeles, CA, USA; ${ }^{3}$ Department of Pathology, Memorial Sloan-Kettering Cancer Center, New York, NY, USA; ${ }^{4}$ Department of Epidemiology and Biostatistics, Memorial Sloan-Kettering Cancer Center, New York, NY, USA and ${ }^{5}$ Department of Medicine, Memorial SloanKettering Cancer Center, New York, NY, USA

B7-H3 and B7x are members of the B7 family of immune regulatory ligands that are thought to attenuate peripheral immune responses through co-inhibition. Previous studies have correlated their overexpression with poor prognosis and decreased tumor-infiltrating lymphocytes in various carcinomas including uterine endometrioid carcinomas, and mounting evidence supports an immuno-inhibitory role in ovarian cancer prognosis. We sought to examine the expression of B7-H3 and B7x in 103 ovarian borderline tumors and carcinomas and study associations with clinical outcome. Using immunohistochemical tissue microarray analysis on tumor specimens, we found that 93 and $100 \%$ of these ovarian tumors express B7-H3 and B7x, respectively, with expression found predominantly on cell membranes and in cytoplasm. In contrast, only scattered B7-H3- and B7x-positive cells were detected in non-neoplastic ovarian tissues. B7-H3 was also expressed in the endothelium of tumor-associated vasculature in $44 \%$ of patients, including $78 \%$ of patients with high-stage tumors (FIGO stages III and IV), nearly all of which were high-grade serous carcinomas, and $26 \%$ of patients with low-stage tumors (FIGO stages I and II; $P<0.001$ ), including borderline tumors. Analysis of cumulative survival time and recurrence incidence revealed that carcinomas with B7-H3-positive tumor vasculature were associated with a significantly shorter survival time $(P=0.02)$ and a higher incidence of recurrence $(P=0.03)$. The association between $\mathrm{B} 7-\mathrm{H} 3$-positive tumor vasculature and poor clinical outcome remained significant even when the analysis was limited to the high-stage subgroup. These results show that ovarian borderline tumors and carcinomas aberrantly express B7-H3 and B7x, and that B7-H3-positive tumor vasculature is associated with high-grade serous histological subtype, increased recurrence and reduced survival. B7-H3 expression in tumor vasculature may be a reflection of tumor aggressiveness and has diagnostic and immunotherapeutic implications in ovarian carcinomas.

Modern Pathology (2010) 23, 1104-1112; doi:10.1038/modpathol.2010.95; published online 21 May 2010

Keywords: B7; co-inhibition; co-stimulation; endothelium; ovarian cancer; serous carcinoma; T cell; tumor vasculature

Correspondence: Dr RA Soslow, MD, Memorial Sloan-Kettering Cancer Center, 1275 York Avenue, New York, NY 10065, USA.

E-mail: soslowr@mskcc.org

${ }^{*}$ Current address: Department of Microbiology and Immuno-

logy, Albert Einstein College of Medicine, Bronx, NY, USA.

'These authors contributed equally to this work.

${ }^{\ddagger}$ Current address: Genentech, Inc., South San Francisco, CA, USA.

Received 17 November 2009; revised 24 February 2010; accepted 1 March 2010; published online 21 May 2010
Ovarian cancer is the leading cause of death from gynecological malignancies, accounting for approximately 15000 deaths in the United States annually. Despite initial response to chemotherapy, with some improvement in overall survival, only a minority of patients survive beyond 5 years. ${ }^{1-3}$

Many recent studies in ovarian cancer have shown that overall survival and recurrence rates are associated with the state of the local adaptive immune response, that is, the presence of 
intratumoral or regulatory $\mathrm{T}$ lymphocytes. ${ }^{4-7}$ The mechanisms underlying this association are unclear; however, tumors may have dynamic interactions with the immune system, expressing tumor-associated antigens and becoming targets for $\mathrm{T}$ cellmediated adaptive immune responses. ${ }^{8}$

T-cell co-stimulation and co-inhibition are primarily generated by interactions between members of the B7 family of immune regulatory ligands and their receptor, CD28. Phylogenetically, the B7 family is divided into three groups. ${ }^{9,10}$ Group I is involved in low-stage $\mathrm{T}$-cell responses and $\mathrm{T}$ - and $\mathrm{B}$-cell interactions in lymphoid tissues. Group II is involved in peripheral immune tolerance and T-cell impairment during chronic viral infections. ${ }^{11,12}$ Group III consists of B7-H3 (B7RP-2) and B7x (B7$\mathrm{H} 4, \mathrm{~B} 7 \mathrm{~S} 1$ ), the newest members of the B7 family, which are thought to attenuate peripheral immune responses through co-inhibition. Their counterreceptors and their precise roles in T-cell regulation remain to be defined; however, they may have a significant role in the interaction between tumors and the immune system.

B7-H3 and B7x mRNA, but not their respective protein products, can be readily detected in most normal human tissues. ${ }^{13-15}$ Recent studies have shown expression of B7-H3 and B7x protein to be associated with aggressive behavior in various genitourinary carcinomas. ${ }^{16-18}$ In addition, a recent study showed that B7x (B7-H4) overexpression correlated inversely with tumor-infiltrating lymphocytes in high-risk uterine endometrioid carcinomas, ${ }^{19}$ and B7x (B7-H4) overexpression has been reported in a cohort of epithelial ovarian tumors. ${ }^{20}$ To our knowledge, B7-H3 expression in ovarian tumors or tumor vasculature has not been examined. We therefore sought to examine the expression of B7-H3 and B7x in ovarian tumors and associated vasculature and their association with clinical outcome because of the link between intratumoral $\mathrm{T}$ lymphocytes and prognosis in ovarian cancer, ${ }^{4-7}$ and the reported downregulation of $\mathrm{T}$ cell-mediated antitumor immunity mediated by B7-H3 and B7x.

\section{Materials and methods}

\section{Tumor Microarrays}

Patients undergoing surgery from 1980 to 2004 for gynecological malignancies at our institution had their tumor specimens banked under an institutional review board (IRB)-approved tissue acquisition protocol after giving their informed consent. Two tumor microarrays were constructed-one from low-stage patients (International Federation of Gynecology and Obstetrics (FIGO) stages IA-IIC) and one from high-stage patients (FIGO stages III and IV). The low-stage microarray was composed of epithelial ovarian tumors from patients undergoing surgery between 1980 and 2000. A total of 63 tumors were included, of which 37 were carcinomas and 26 were borderline tumors. Of the carcinomas, 17 were either pure serous carcinomas or mixed epithelial carcinomas with a serous component, 9 were endometrioid adenocarcinomas, and 11 were clear cell carcinomas. There were 6 serous borderline tumors, 7 endometrioid borderline tumors, and 13 intestinal mucinous borderline tumors. The highstage array was composed of 68 tumors from 40 patients undergoing surgery from 1989 to 2004. Primary, metastatic, and recurrent tumors were included in this tissue microarray. All high-stage tumors except for one were high-grade serous or mixed epithelial carcinomas; the remaining tumor was a clear cell carcinoma. All cases were reviewed by a gynecologic pathologist (RAS), and final pathologic diagnoses were confirmed. Histological subtype, assigned following World Health Organization criteria, Shimizu-Silverberg grade, FIGO stage, age, and survival data were recorded.

Tissue microarrays were constructed by obtaining mostly triplicate cores of formalin-fixed paraffinembedded tumor specimens and re-embedding the cores in an arrayed master block with techniques developed at our institution and others. ${ }^{16,17}$ The Beecher Instruments (Sun Prairie, WI, USA) arraying device was used to produce sample circular cores $0.6 \mathrm{~mm}$ in diameter. Whole sections, formalinfixed paraffin-embedded, from randomly collected ovaries lacking neoplasms were selected for immunohistochemistry.

\section{Immunohistochemical Methods}

The methodology has been published elsewhere. ${ }^{16}$ Antibodies used in this study included goat antiB7-H3 (catalog no. BAF1027; R\&D Systems; Minneapolis, MN, USA), goat anti-B7x (Clone H74; eBioscience, San Diego, CA, USA), and isotype control antibodies (R\&D Systems). Positive and negative controls, as determined by flow cytometry, were cell lines MDA-MB-468 (B7-H3 negative and B7x positive), MDA-MB-231 (B7-H3 negative, B7x negative, and B7-H1 positive), and B7-H3/BWZ (B7-H3 positive and B7x negative), which was transfected to express B7-H3. Immunohistochemical staining of B7-H3 and B7x was performed using the EnVision Peroxidase Detection System and DAKO TechMate 500/1000 staining machine (BioTek Solutions). Sections $(4 \mu \mathrm{m})$ were mounted on Superfrost plus slides, deparaffinized, rehydrated, incubated with Dako Target Retrieval Solution $(\mathrm{pH}$ 9), and heated at $900 \mathrm{~W}$ for $2 \mathrm{~min}$. The sections were incubated with anti-B7-H3 $(5 \mu \mathrm{g} / \mathrm{ml})$ and anti-B7x $(10 \mu \mathrm{g} / \mathrm{ml})$ and further processed using the EnVision Peroxidase Detection System with biotinylated secondary antibodies. The sections were counterstained with Mayer's hematoxylin solution, and coverslips were applied. Irrelevant isotype-matched antibodies were used to control for nonspecific staining. 


\section{Immunohistochemical Evaluation}

B7-H3 and B7x expression data were limited to tumors with sufficient tissue for immunohistochemical evaluation. A semiquantitative scoring scheme based on the distribution of positive tumor cells and the staining intensity was used to evaluate the expression of $\mathrm{B} 7-\mathrm{H} 3$ and $\mathrm{B} 7 \mathrm{x}$. The distribution score, which is an estimate of the percentage of positively stained cells, was multiplied by an intensity factor, which ranged from 1 (staining intensity just exceeding background) to 3 (dark brown staining obvious on macroscopic inspection of the slide). This yielded the immunohistochemical score, ${ }^{18}$ ranging from 0 to 300 . Expression of B7-H3 and B7x was also assessed in the endothelium of any readily identified vasculature present in core tissue with overlying or adjacent tumor cells.

\section{Statistical Analysis}

Statistical analysis was performed using R software (freeware, R-project). B7-H3 and B7x levels were compared between groups using the Kruskal-Wallis test and Fisher's exact test, where appropriate. Cumulative survival time was calculated using the Kaplan-Meier method and analyzed using the logrank test. Recurrence was treated in a competing risks model, using Gray's statistic to analyze. All borderline tumors were excluded in analyses comparing grade, stage, and clinical outcome. Two-sided $P$-values of $<0.05$ were deemed significant. The association between tumor vascularization (positive vs negative) and overall survival or recurrence was analyzed as a single variable, unadjusted for other variables; it was evaluated for all patients (tumors) and for the subset of advanced disease (stages III and IV).

\section{Results}

\section{Study Population and Clinical Outcome}

The clinical and pathological features of the patients are summarized in Table 1. An association between histological subtype and stage was evident. All patients with borderline tumors and endometrioid carcinomas had low-stage disease and all but one of the clear cell carcinoma patients had low-stage disease as well. In all $76 \%$ of serous carcinoma patients had high-stage disease. For low-stage patients, the median follow-up was 8 years (range 0.6-25.5), whereas the median survival was not reached. For high-stage patients, the median followup was 1 year (range 0.4-7.9) and the median survival was 4.3 years (95\% CI 3.7-5.5). Tumor stage $(P<0.001)$ and grade $(P=0.03)$ correlated well with overall survival.
Table 1 Clinical and pathological features

\begin{tabular}{|c|c|c|c|c|}
\hline & \multirow[b]{2}{*}{$\begin{array}{c}\text { All } \\
\text { patients }\end{array}$} & \multicolumn{3}{|c|}{ Clinical characteristics } \\
\hline & & $\begin{array}{c}\text { Border- } \\
\text { line }^{\mathrm{a}}\end{array}$ & $\begin{array}{c}\text { Low- } \\
\text { stage } C A^{\mathrm{b}}\end{array}$ & $\begin{array}{c}\text { High- } \\
\text { stage } C A^{\mathrm{b}}\end{array}$ \\
\hline Number & 103 & 26 & 37 & 40 \\
\hline \multicolumn{5}{|l|}{ Vital status } \\
\hline Alive & 57 & 19 & 28 & 30 \\
\hline Dead & 43 & 5 & 8 & 10 \\
\hline \multicolumn{5}{|l|}{ Age } \\
\hline Mean (median) & $60.4(60)$ & - & - & $60.4(60)$ \\
\hline Range & $36-79$ & & & $36-79$ \\
\hline \multicolumn{5}{|l|}{ Histology } \\
\hline Borderline tumors $^{\mathrm{a}}$ & 26 & 26 & 0 & 0 \\
\hline Clear cell CA & 12 & 0 & 11 & 1 \\
\hline Endometrioid CA & 9 & 0 & 9 & 0 \\
\hline Serous CA & 45 & 0 & 11 & 34 \\
\hline Mixed epithelial CA & 11 & 0 & 6 & 5 \\
\hline \multicolumn{5}{|l|}{ Grade } \\
\hline 1 & 5 & & 5 & 0 \\
\hline 2 & 23 & & 19 & 4 \\
\hline 3 & 48 & & 13 & 35 \\
\hline
\end{tabular}

CA, carcinoma.

${ }^{\mathrm{a}}$ Serous $(n=6)$, endometrioid $(n=7)$, and intestinal mucinous $(n=13)$ borderline tumors.

${ }^{\mathrm{b}}$ Low stage is FIGO stages I and II and high stage is FIGO stages III and IV.

\section{Ovarian Epithelial Tumors Overexpress B7-H3 and $B 7 x$}

B7-H3 expression by immunohistochemistry was present in $96(93 \%)$ of 103 ovarian tumors (Figures 1a-c). Mean levels of B7-H3 staining ranged from 116.2 to 153.6, depending on histological subtype (range 0-280). B7x positivity was present in all 103 ovarian tumors (Figures 2a-c). Mean levels of B7x staining ranged from 168.8 to 226.4, depending on histological subtype (range 20-300). In contrast, no significant B7-H3 or B7x expression was found in 14 non-neoplastic ovarian specimens (Figures 1d and 2d); only a few scattered B7-H3- and B7x-positive stromal cells were detected. Membranous and cytoplasmic staining were the two most frequently observed patterns of B7-H3 and B7x protein expression. In addition, some cases showed a dot-like staining pattern for B7x as well, suggesting B7x may localize within the Golgi apparatus (Figure 2). We observed tumor-infiltrating lymphocytes in some tumors, but the lymphocytes were B7-H3 and B7x negative.

There was no difference in either B7-H3 or B7x expression between different histological subtypes. We excluded borderline tumors from the cohort and then analyzed 77 carcinomas. Kruskal-Wallis analysis showed statistically significant correlations between expression of B7-H3 and B7x and tumor stage $(P=0.004$ and 0.007 , respectively $)$, but not with grade. 

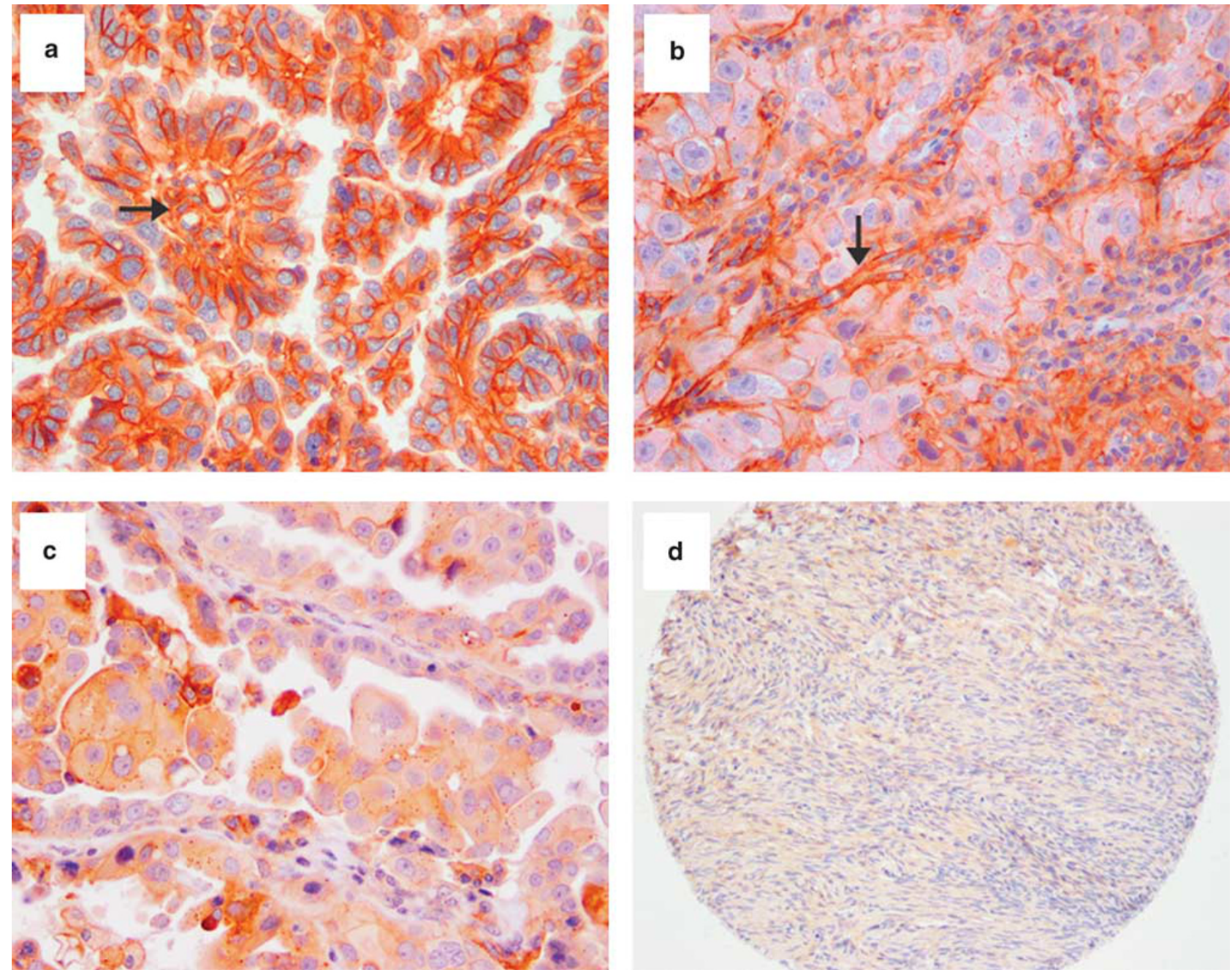

Figure 1 Ovarian tumor tissue microarray immunohistochemical staining of B7-H3. B7-H3 expression is shown in serous cell carcinoma and tumor vessels $(\mathbf{a}, \times 200)$, in clear cell carcinoma and tumor vessels $(\mathbf{b}, \times 200)$, and in serous carcinoma but not in tumor vessels $(\mathbf{c}, \times 200)$. No significant B7-H3 staining is observed in non-neoplastic ovarian tissue $(\mathbf{d}, \times 100)$. Arrows represent B7-H3-positive endothelium in tumor-associated vessels.

\section{B7-H3 and B7x Tumor Expression Correlates with Recurrence and Survival}

We analyzed prognostic significance by comparing carcinomas with high B7-H3 levels $(>50)$ to those with low B7-H3 expression levels $(\leq 50)$ (Table 2); levels were chosen empirically. High B7-H3 expression was associated with increased recurrence (51 vs $39 \%$ in low B7-H3 carcinomas) and mortality (45 vs $38 \%$ in low B7-H3 carcinomas), although the differences did not reach statistical significance.

We studied the prognostic significance of $\mathrm{B} 7 \mathrm{x}$ expression in carcinomas by comparing a B7x highexpression group ( $>100$ ) with a B7x low-expression group $(\leq 100)$; levels were chosen empirically. Carcinomas with high B7x expression were associated with increased recurrence (53 vs $27 \%$ in low B7x carcinomas) and mortality (45 vs $36 \%$ in low B7x carcinomas), although the differences did not reach statistical significance.

\section{B7-H3 Expression in Tumor Vasculature is Associated with Histology and Stage}

We analyzed B7-H3 expression in the endothelium of tumor-associated vasculature with specimens from 93 borderline tumor and carcinoma patients whose tumors showed recognizable tumor vasculature on core tissue (Table 3 and Figure 1). In all, 41 $(44 \%)$ cases showed B7-H3 expression in tumor vasculature. Fisher's exact test analysis showed that there was an association between B7-H3 expression in tumor vasculature and histological type $(P<0.001$; Table 3 and Figure 3$)$. Serous carcinomas had a higher percentage of B7-H3positive tumor vasculature $(62 \%(28 / 45))$ compared with borderline tumors of all types (15\% (4/26)) and clear cell and endometrioid carcinomas.

There was a statistically significant association $(P<0.001)$ between stage and B7-H3 expression in tumor vasculature (Table 3 and Figure 3). A majority of high-stage (FIGO stages III and IV) tumors had 

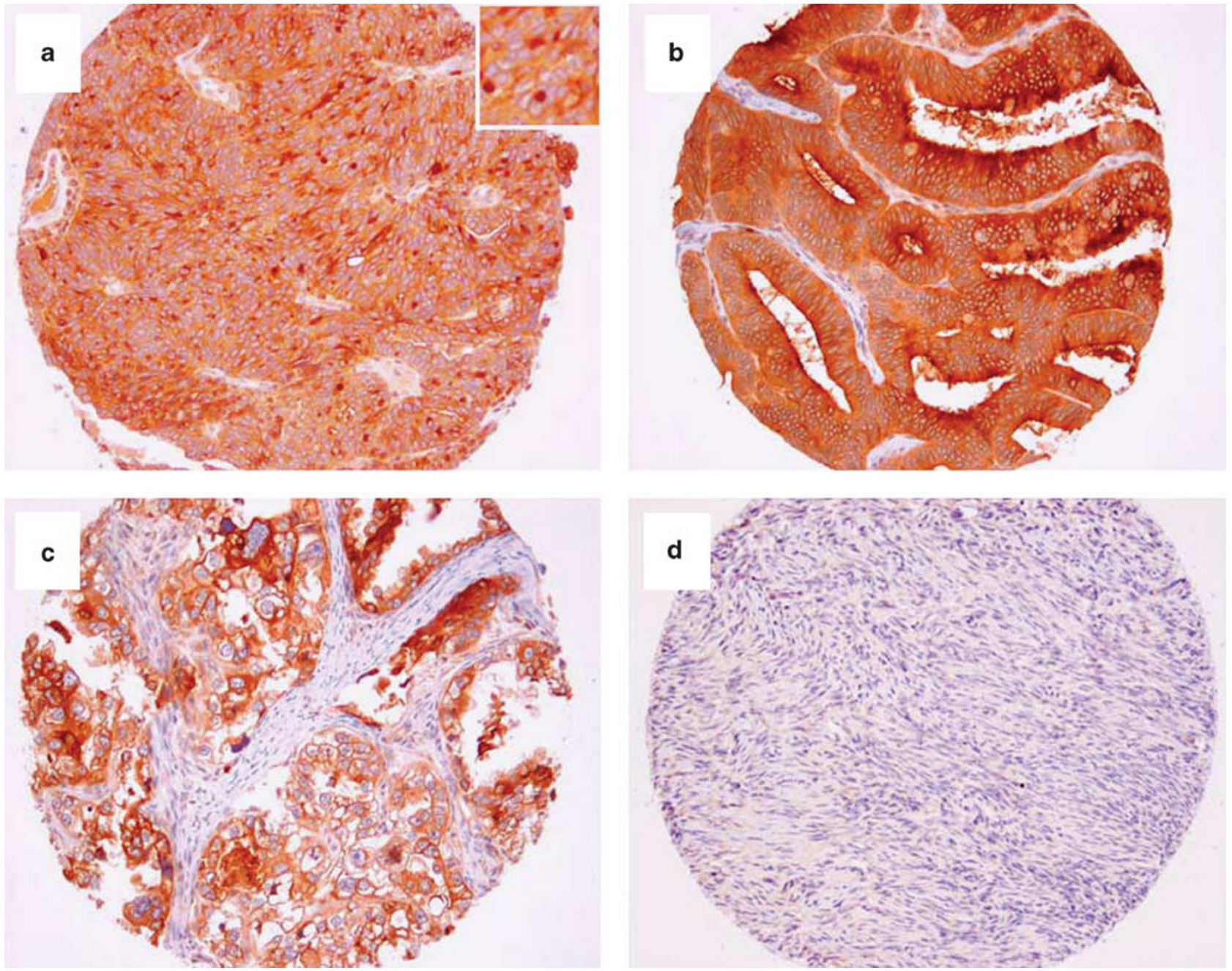

Figure 2 Ovarian tumor tissue microarray immunohistochemical staining of B7x. B7x expression is shown in serous carcinoma $(\mathbf{a}, \times 100)$, in endometrioid carcinoma $(\mathbf{b}, \times 100)$, and in clear cell carcinoma $(\mathbf{c}, \times 100)$. B7x staining is both cytoplasmic and membranous (a-c). Some tumors showed a dot-like staining pattern (inset) (a). No significant B7x expression was observed in nonneoplastic ovarian tissues $(\mathbf{d}, \times 60)$ or vasculature.

Table 2 Relationship between B7-H3 and B7x expression in carcinomas and overall survival and recurrence

\begin{tabular}{cccccccc}
\hline & & \multicolumn{3}{c}{ Overall survival } & & \multicolumn{2}{c}{ Recurrence } \\
\cline { 3 - 4 } Factor & $\begin{array}{c}\text { No. of } \\
\text { patients }\end{array}$ & $\begin{array}{c}\text { No. of } \\
\text { deaths }\end{array}$ & $\begin{array}{c}\text { Median } \\
\text { (month) }\end{array}$ & $\begin{array}{c}\text { P- } \\
\text { value }\end{array}$ & $\begin{array}{c}\text { No. of } \\
\text { vecurrences }\end{array}$ & $\begin{array}{c}\text { P- } \\
\text { value }\end{array}$ \\
\hline B7-H3 & & & & & & \\
$\leq 50$ & 13 & 5 & 75.3 & 0.64 & 5 & 0.96 \\
$>50$ & 64 & 29 & 84.7 & & 33 & \\
$B 7 x$ & & & & & & \\
$\leq 100$ & 11 & 4 & 57.4 & 0.80 & 3 & 0.38 \\
$>100$ & 66 & 30 & 80.2 & & 35 & \\
\hline
\end{tabular}

B7-H3-positive tumor vasculature (78\% (25/32)). In contrast, a minority of low-stage (FIGO stages I and II) tumors had B7-H3-positive tumor vasculature $(26 \%(16 / 61))$.
When borderline tumors were excluded from the analysis, the association between $\mathrm{B} 7-\mathrm{H} 3$ expression in tumor vasculature and stage was still statistically significant $(P<0.001$; Table 3$)$. There was no significant association between B7-H3 expression in tumor vasculature and tumor grade. Unlike B7$\mathrm{H} 3$, B7x expression in tumor-associated endothelium was not observed.

\section{B7-H3 Expression in Tumor Vasculature of Ovarian Carcinomas is Associated with Poor Survival}

We further analyzed whether there was an association between survival and B7-H3 expression in tumor vasculature. Cumulative survival time was calculated using the Kaplan-Meier method and analyzed using the log-rank test. This showed that carcinoma patients with B7-H3-positive vasculature had significantly shorter survival times $(P=0.02)$. Only 54 and 
Table 3 Relationships between B7-H3 in tumor vasculature and clinicopathological variables

\begin{tabular}{|c|c|c|c|c|c|c|}
\hline \multirow[b]{2}{*}{ Factor } & \multirow[b]{2}{*}{ Tуре } & \multicolumn{2}{|c|}{ Stage $^{\mathrm{a}}$} & \multicolumn{3}{|c|}{ Tumor vasculature B7-H3 } \\
\hline & & Low & High & Positive & Negative & $\mathrm{P}$-value \\
\hline $\begin{array}{l}\text { Histology } \\
(N=103)\end{array}$ & $\begin{array}{l}\text { Borderline tumor } \\
\text { Clear cell CA } \\
\text { Endometrioid CA } \\
\text { Serous CA } \\
\text { Mixed epith CA }\end{array}$ & $\begin{array}{r}26 \\
11 \\
9 \\
11 \\
6\end{array}$ & $\begin{array}{r}0 \\
1 \\
0 \\
34 \\
5\end{array}$ & $\begin{array}{r}4 \\
5 \\
1 \\
28 \\
3\end{array}$ & $\begin{array}{r}20 \\
7 \\
8 \\
12 \\
5\end{array}$ & $<0.001$ \\
\hline Tumor vasculature $(N=93)$ & $\begin{array}{l}\text { Low stage } \\
\text { High stage }^{\mathrm{a}}\end{array}$ & $\frac{61}{-}$ & $\overline{32}$ & $\begin{array}{l}16 \\
25\end{array}$ & $\begin{array}{r}45 \\
7\end{array}$ & $<0.001$ \\
\hline $\begin{array}{l}\text { Stage }^{\mathrm{b}} \\
(N=76)\end{array}$ & $\begin{array}{l}\text { I } \\
\text { II } \\
\text { III } \\
\text { IV }\end{array}$ & $\begin{array}{r}21 \\
17 \\
0 \\
0\end{array}$ & $\begin{array}{r}0 \\
0 \\
31 \\
7\end{array}$ & $\begin{array}{r}7 \\
6 \\
18 \\
5\end{array}$ & $\begin{array}{r}14 \\
11 \\
7 \\
0\end{array}$ & $<0.001$ \\
\hline $\begin{array}{l}\text { Grade }^{\mathrm{b}} \\
(N=76)\end{array}$ & $\begin{array}{l}1 \\
2 \\
3\end{array}$ & $\begin{array}{r}5 \\
19 \\
13\end{array}$ & $\begin{array}{r}0 \\
4 \\
35\end{array}$ & $\begin{array}{r}2 \\
8 \\
27\end{array}$ & $\begin{array}{r}3 \\
13 \\
16\end{array}$ & 0.15 \\
\hline
\end{tabular}

CA, carcinoma.

${ }^{\mathrm{a}}$ Low stage is FIGO stages I and II and high stage is FIGO stages III and IV.

${ }^{\mathrm{b}}$ Carcinomas only.

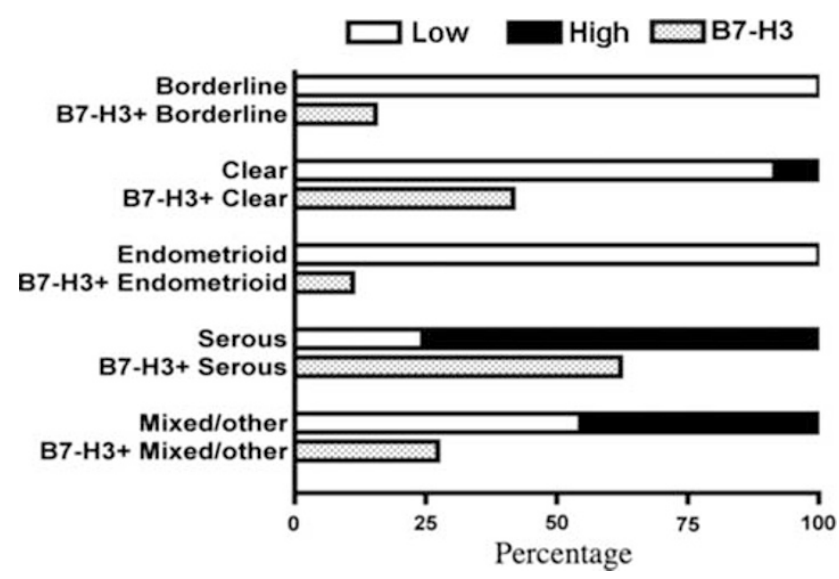

Figure 3 Distribution of tumor vasculature B7-H3 expression by different histological subtypes (borderline tumors, clear cell, endometrioid, serous, and mixed carcinomas) and ovarian tumor stages (low vs high stage). The top line represents the percentage of cases in each stage category. The bottom line represents the percentage with B7-H3-positive vasculature.

$36 \%$ of carcinoma patients with B7-H3-positive tumor vasculature were alive compared with 87 and $65 \%$ of carcinoma patients with B7-H3-negative tumor vasculature at 50 and 100 months after diagnosis, respectively (Figure 4a). We further stratified patients with high stage (FIGO stages III and IV) carcinomas into two groups based on B7-H3 expression in tumor vasculature. Outcome data were still significantly different for these two groups. At 50 months after diagnosis, only $45 \%$ of the B7-H3-positive group was still alive versus $80 \%$ of the B7-H3-negative group $(P=0.02$; Figure $4 \mathrm{~b})$. Therefore, B7-H3 expression in tumor vasculature is associated with poor survival in high-stage ovarian cancer patients.

\section{B7-H3 Expression in Tumor Vasculature of Ovarian Carcinomas is Associated with Cumulative Recurrence Incidence}

We also assessed whether there was an association between cumulative recurrence incidence and B7H3 expression in tumor vasculature. Competing risk models with Gray's statistical analysis revealed that carcinoma patients with B7-H3-positive tumor vasculature had a significantly higher cumulative recurrence incidence than carcinoma patients with B7-H3-negative tumor vasculature $(P=0.03)$. At 50 months after diagnosis, the overall cumulative recurrence incidence in the B7-H3-positive tumor vasculature group was twice that of the B7-H3negative group ( 60 vs $30 \% ; P=0.03$; (Figure 5a). We further stratified high-stage individuals by B7-H3 expression in tumor vasculature. At 50 months after diagnosis of high-stage ovarian carcinoma, the cumulative incidence of recurrence was $84 \%$ in patients with B7-H3-positive tumor vasculature compared with $40 \%$ in patients with B7-H3-negative tumor vasculature $(P=0.11$; Figure $5 \mathrm{~b})$. Therefore, there is a trend toward significance, suggesting an association between B7-H3 expression in tumor vasculature and cumulative incidence of recurrence in high-stage ovarian cancer.

\section{Discussion}

Recent data show increasing evidence of the role that immune regulation has in ovarian carcinogenesis. Zhang et $a l^{6}$ showed the association between tumor-infiltrating lymphocytes and favorable outcome in ovarian cancer patients. Further studies support this finding ${ }^{6,5}$ and have shown that 

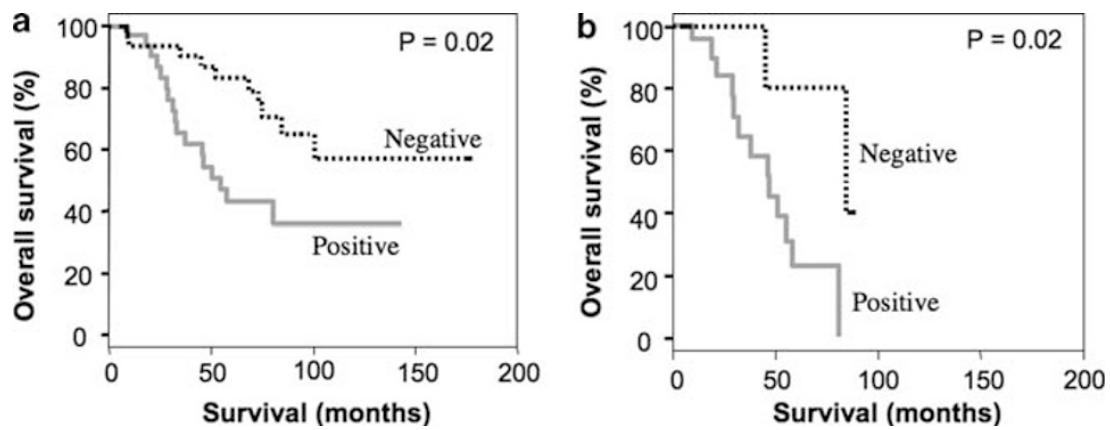

Figure 4 Kaplan-Meier overall survival curves for carcinoma patients with (red solid line) and without (black dotted line) B7-H3positive tumor vasculature. Analysis for all patients (a) and for high-stage (FIGO stages III and IV) patients (b). Log-rank statistical test shows $P=0.02$ for all patients and high-stage patients. The color reproduction of this figure is available on the html full text version of the manuscript.
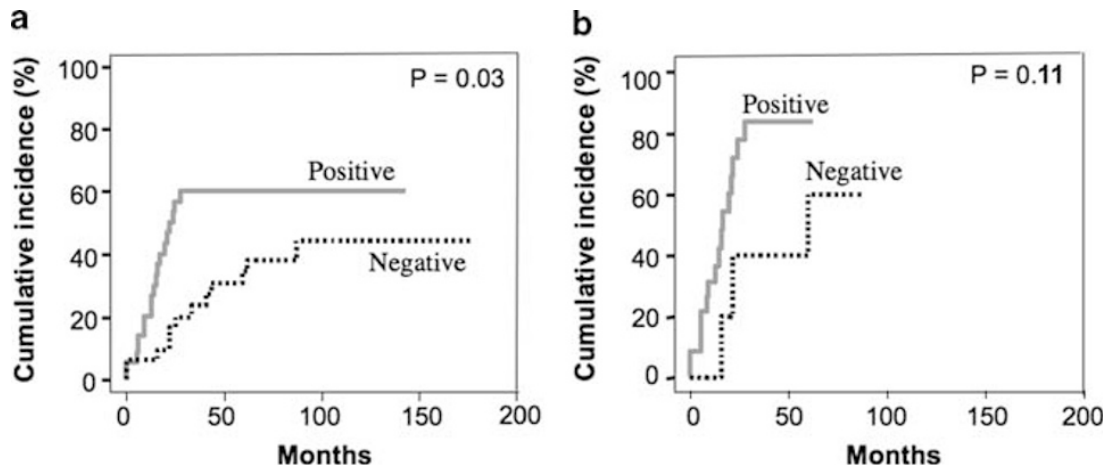

Figure 5 Kaplan-Meier curves showing the cumulative incidence of recurrence in carcinoma patients with (red) and without (black) B7-H3-positive tumor vasculature. Competing risks models with Gray's statistic analysis in all patients (a) and in high-stage patients (b). Log-rank statistical test shows $P=0.03$ for all patients and $P=0.11$ for high-stage patients. The color reproduction of this figure is available on the html full text version of the manuscript.

this association may also be related to BRCA1 deficiency. ${ }^{7}$

T-cell co-stimulation and co-inhibition is primarily generated by the interaction between members of the B7 family of immune regulatory ligands and their receptor, CD28, which may have a significant role in the interaction between tumors and the immune system. B7-H3 and B7x (B7-H4), the newest members of the B7 family, are thought to attenuate peripheral immune responses through co-inhibition of T-cell function. ${ }^{9,13,21-24}$ B7-H3 and B7x mRNA, but not their respective protein products, can be detected in most normal human tissues. ${ }^{13-15}$ B7-H3 protein expression has been reported in normal placental tissue, ${ }^{25}$ whereas both $\mathrm{B} 7-\mathrm{H} 3$ and $\mathrm{B} 7 \mathrm{x}$ expression have been reported in various genitourinary carcinomas and associated tumor vasculature. ${ }^{16-18}$ Interestingly, B7x (B7-H4) overexpression was reported in high-risk uterine endometrioid adenocarcinomas and correlated inversely with tumor-infiltrating lymphocytes, ${ }^{19}$ and B7x (B7-H4) overexpression has been reported in a cohort of epithelial ovarian tumors. ${ }^{20}$ To our knowledge, B7$\mathrm{H} 3$ expression in ovarian tumors or tumor vascu- lature has not been examined. We postulated that ovarian tumor development and progression may be associated with downregulation of T cell-mediated antitumor immunity through B7x and B7-H3. We sought to examine the expression of $\mathrm{B} 7-\mathrm{H} 3$ and $\mathrm{B} 7 \mathrm{x}$ in ovarian tumors and their association with clinical outcome.

We found that most ovarian cancers express B7H3 and B7x. In line with past studies, ${ }^{13-15}$ only few scattered stromal cells in non-neoplastic ovarian tissues expressed B7-H3 and B7x. We also found that B7-H3 was expressed in a significant proportion of tumor-associated vascular endothelial cells. This finding is similar to that of another study reporting B7-H3 expression in renal cell carcinoma tumor vasculature, ${ }^{17}$ but we did not observe B7x expression in tumor vasculature, which contrasts with a previous report of significant B7x positivity in renal cell carcinoma tumor vasculature. ${ }^{18}$ This discrepancy may be due to vascular heterogeneity among different types of tumors.

One of the most noteworthy findings in this study is that B7-H3 expression in tumor vasculature was associated with histological subtype, stage, 
cumulative recurrence incidence, and poor clinical outcome. The association of B7-H3 tumor vasculature expression with poor clinical outcome remained significant even in patients with high-stage tumors. Other studies have reported an association between B7-H3 expression and survival in renal cell carcinomas (expression in both tumor cells and tumor vasculature) ${ }^{17}$ and in prostate carcinomas (expression in tumor cells only). ${ }^{16}$

It is unclear whether the prognostic value of B7-H3 tumor vasculature expression is intrinsically related to high-stage ovarian tumors, high-grade tumors, or serous carcinomas in general because high-grade and high-stage ovarian tumors tend to be serous by histology. ${ }^{26,27}$ However, it is important to note that a trend toward significance of B7-H3 expression in tumor vasculature remains, even in a smaller cohort of high-stage serous carcinomas. Interestingly, a previous study linking tumor-infiltrating lymphocytes to favorable outcome showed that the association was restricted to serous carcinomas. ${ }^{7}$

It is currently not known what signals trigger B7H3 expression in tumor vessels, but mounting evidence suggests there may be a significant connection between tumor angiogenesis and $\mathrm{T}$ cellmediated immune regulation. In general, vascular endothelial growth factor, matrix metalloproteinases, and prostaglandin-endoperoxide synthases have vital roles in tumor angiogenesis. ${ }^{28-31}$ It is interesting to note that a recent study found a correlation between these molecules and B7-H3 at the mRNA level in colorectal tumors, ${ }^{32}$ suggesting that B7-H3 may be involved in the process of tumor angiogenesis. Furthermore, a recent study showed that the absence of intratumoral $\mathrm{T}$ cells was associated with poor outcome and increased levels of vascular endothelial growth factor. ${ }^{4}$

As mentioned above, tumor-infiltrating lymphocytes in ovarian tumors have been reported in association with BRCA-deficient patients and favorable outcome. ${ }^{7}$ It is interesting to speculate whether B7-H3 expression in tumor vasculature may be a reflection of BRCA1 function. There is also potential for B7-H3 to be used as a therapeutic target in ovarian tumors. Treatment may have a dual effect of enhanced T cell-mediated antitumor immunity (immunotherapy) and destruction of tumor vessels (antiangiogenesis). In fact, early results from a recent study using a monoclonal antibody specific for B7H3 have shown promise in the treatment of solid tumors metastatic to the central nervous system. ${ }^{33}$ Finally, B7-H3 protein expression has the potential to be used both as a diagnostic and prognostic aid for ovarian tumors, and perhaps to be used in future surgical decision making. ${ }^{34}$ Further studies are warranted to explore these possibilities.

\section{Disclosure/conflict of interest}

Dr Jakob Dupont presently works at Genentech.

\section{References}

1 Cannistra SA. Cancer of the ovary. N Engl J Med 2004:351:2519-2529.

2 Jemal A, Siegel R, Hao Y, et al. Cancer statistics, 2009. CA Cancer J Clin 2009;59:225-249.

3 Ozols RF. Systemic therapy for ovarian cancer: current status and new treatments. Semin Oncol 2006;33: S3-S11.

4 Zhang L, Conejo-Garcia JR, Katsaros D, et al. Intratumoral $\mathrm{T}$ cells, recurrence, and survival in epithelial ovarian cancer. N Engl J Med 2003;348:203-213.

5 Curiel TJ, Coukos G, Zou L, et al. Specific recruitment of regulatory $\mathrm{T}$ cells in ovarian carcinoma fosters immune privilege and predicts reduced survival. Nat Med 2004;10:942-949.

6 Sato E, Olson SH, Ahn J, et al. Intraepithelial CD8+ tumor-infiltrating lymphocytes and a high CD8+/ regulatory $\mathrm{T}$ cell ratio are associated with favorable prognosis in ovarian cancer. Proc Natl Acad Sci USA 2005;102:18538-18543.

7 Clarke B, Tinker AV, Lee CH, et al. Intraepithelial T cells and prognosis in ovarian carcinoma: novel associations with stage, tumor type, and BRCA1 loss. Mod Pathol 2009;22:393-402.

8 Rosenberg SA. Progress in human tumour immunology and immunotherapy. Nature 2001;411:380-384.

9 Zang X, Loke P, Kim J, et al. B7x: a widely expressed B7 family member that inhibits T cell activation. Proc Natl Acad Sci USA 2003;100:10388-10392.

10 Zang X, Allison JP. The B7 family and cancer therapy: costimulation and coinhibition. Clin Cancer Res 2007;13(18 Pt 1):5271-5279.

11 Okazaki T, Honjo T. The PD-1-PD-L pathway in immunological tolerance. Trends Immunol 2006; 27:195-201.

12 Barber DL, Wherry EJ, Masopust D, et al. Restoring function in exhausted CD8 T cells during chronic viral infection. Nature 2006;439:682-687.

13 Sica GL, Choi IH, Zhu G, et al. B7-H4, a molecule of the B7 family, negatively regulates $\mathrm{T}$ cell immunity. Immunity 2003;18:849-861.

14 Tringler B, Zhuo S, Pilkington G, et al. B7-h4 is highly expressed in ductal and lobular breast cancer. Clin Cancer Res 2005;11:1842-1848.

15 Chapoval AI, Ni J, Lau JS, et al. B7-H3: a costimulatory molecule for T cell activation and IFN-gamma production. Nat Immunol 2001;2:269-274.

16 Zang X, Thompson RH, Al-Ahmadie HA, et al. B7-H3 and $\mathrm{B} 7 \mathrm{x}$ are highly expressed in human prostate cancer and associated with disease spread and poor outcome. Proc Natl Acad Sci USA 2007;104: 19458-19463.

17 Crispen PL, Sheinin Y, Roth TJ, et al. Tumor cell and tumor vasculature expression of B7-H3 predict survival in clear cell renal cell carcinoma. Clin Cancer Res 2008;14:5150-5157.

18 Krambeck AE, Thompson RH, Dong H, et al. B7-H4 expression in renal cell carcinoma and tumor vasculature: associations with cancer progression and survival. Proc Natl Acad Sci USA 2006;103: 10391-10396.

19 Miyatake T, Tringler B, Liu W, et al. B7-H4 (DD-O110) is overexpressed in high risk uterine endometrioid adenocarcinomas and inversely correlated with tumor T-cell infiltration. Gynecol Oncol 2007;106: $119-127$. 
20 Tringler B, Liu W, Corral L, et al. B7-H4 overexpression in ovarian tumors. Gynecol Oncol 2006;100:44-52.

21 Prasad DV, Richards S, Mai XM, et al. B7S1, a novel B7 family member that negatively regulates $\mathrm{T}$ cell activation. Immunity 2003;18:863-873.

22 Ling V, Wu PW, Spaulding V, et al. Duplication of primate and rodent B7-H3 immunoglobulin V-and Clike domains: divergent history of functional redundancy and exon loss. Genomics 2003;82:365-377.

23 Prasad DV, Nguyen T, Li Z, et al. Murine B7-H3 is a negative regulator of $\mathrm{T}$ cells. J Immunol 2004;173: 2500-2506.

24 Suh WK, Gajewska BU, Okada H, et al. The B7 family member B7-H3 preferentially down-regulates T helper type 1-mediated immune responses. Nat Immunol 2003;4:899-906.

25 Petroff MG, Kharatyan E, Torry DS, et al. The immunomodulatory proteins B7-DC, B7-H2, and B7$\mathrm{H} 3$ are differentially expressed across gestation in the human placenta. Am J Pathol 2005;167:465-473.

26 Gilks CB, Ionescu DN, Kalloger SE, et al. Tumor cell type can be reproducibly diagnosed and is of independent prognostic significance in patients with maximally debulked ovarian carcinoma. Hum Pathol 2008;39:1239-1251.
27 Soslow RA. Histologic subtypes of ovarian carcinoma: an overview. Int J Gynecol Pathol 2008;27:161-174.

28 Ruoslahti E. Specialization of tumour vasculature. Nat Rev Cancer 2002;2:83-90.

29 Ruoslahti E, Rajotte D. An address system in the vasculature of normal tissues and tumors. Annu Rev Immunol 2000;18:813-827.

30 Ferrara N. VEGF and the quest for tumour angiogenesis factors. Nat Rev Cancer 2002;2:795-803.

31 Handsley MM, Edwards DR. Metalloproteinases and their inhibitors in tumor angiogenesis. Int J Cancer 2005;115:849-860.

32 Galon J, Costes A, Sanchez-Cabo F, et al. Type, density, and location of immune cells within human colorectal tumors predict clinical outcome. Science 2006;313: 1960-1964.

$33 \mathrm{Xu} \mathrm{H}$, Cheung IY, Guo HF, et al. MicroRNA miR-29 modulates expression of immunoinhibitory molecule B7-H3: potential implications for immune based therapy of human solid tumors. Cancer Res 2009;69: 6275-6281.

34 Adams SF, Levine DA, Cadungog MG, et al. Intraepithelial T cells and tumor proliferation: impact on the benefit from surgical cytoreduction in advanced serous ovarian cancer. Cancer 2009;115:2891-2902. 